\title{
POPULAÇÃO EM SITUAÇÃO DE RUA: MAIOR VULNERABILIDADE E INVISIBILIDADE DURANTE A PANDEMIA DE COVID-19
}

\author{
Amanda Márcia dos Santos Reinaldo' \\ ORCID: 0000-0003-0283-2313 \\ Sandra Cristina Pillon" \\ ORCID: 0000-0001-8902-7549 \\ Cristopher Wagstaff"' \\ ORCID: 0000-0002-8013-1195 \\ Belisa Vieira Silveira" \\ ORCID: 0000-0002-5966-8537
}

Natália de Magalhães Ribeiro Gomes' ORCID: 0000-0003-1717-8692

Maria Odete Pereira'

ORCID: 0000-0002-9418-2524

'Universidade Federal de Minas Gerais. Belo Horizonte, Minas Gerais, Brasil.

"Universidade de São Paulo. Ribeirão Preto, São Paulo, Brasil.

I'University of Birmingham. Birmingham, England, UK.

Autor Correspondente: Maria Odete Pereira E-mail:m.odetepereira@gmail.com

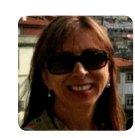

Como citar:

Reinaldo MAS, Pillon SC, Wagstaff C, Silveira BV Gomes NMR, Pereira MO. População em situação de rua: maior vulnerabilidade e invisibilidade durante a pandemia de COVID-19. In: Esperidião E, Saidel MGB

(Orgs.). Enfermagem em saúde mental e COVID-19. 2.ed.rev. Brasília, DF: Editora ABEn; 2020.

p. 45-50. (Série Enfermagem e Pandemias, 4). https://doi.org/10.51234/aben.20.e04.c06

\section{INTRODUÇÃO}

A falta de moradia é uma questão social multifacetada e complexa que afeta milhões de pessoas no mundo. Em geral está associada às condições de vida e a situações de pobreza, um fenômeno real que ocorre independente da renda per capita dos países, que fragiliza a qualidade de vida e bem-estar das pessoas; aumenta a violência urbana; influencia os indicadores econômicos das nações, entre outros fatores imensuráveis e que não se têm pesquisas acerca de políticas eficazes, para mitigar esse problema ${ }^{(1-3)}$.

No ano de 2020, a Organização Mundial de Saúde ${ }^{(1)}$ decretou estado de pandemia associada à rápida contaminação e disseminação da COVID-19 pelo mundo. À medida que a pandemia avançava pelos continentes, a doença expôs a fragilidade dos poderes públicos frente à questão do isolamento e distanciamento social de quem vive em situação de rua, e a dificuldade em manter em funcionamento a assistência oferecida pelos serviços de saúde, incluindo essa população por seus elevados níveis de vulnerabilidade e risco de contaminação.

Nos Países envolvidos na pandemia, o isolamento social prevaleceu como medida de prevenção para disseminação do vírus, mas as pessoas em situação de rua iriam para onde? Alguns Países se organizaram para oferecer locais, como albergues; hotéis e moradias de proteção, onde essas pessoas poderiam ter acesso à alimentação; local para dormir e higiene pessoal, somadas às ações de prevenção ao contágio e disseminação do vírus, por meio da educação em saúde e oferta de insumos de proteção. Outros Países, por diferentes questões, não adotaram políticas públicas para essa população, tornando-a duplamente invisível(4)

No Brasil, não há uma estimativa oficial da população em situação de rua, o que chama a atenção para a invisibilidade do problema para o Estado e dificulta o acesso à seguridade social, corroborando a inexistência de políticas públicas voltadas a mesma. Em 2015, o número estimado 
de pessoas vivendo em situação de rua foi de 101.854. Os dados foram compilados de diferentes Bases de dados, que não se cruzam, dificultando o dimensionamento real do número de cidadãos brasileiros ou imigrantes, que vivem nessas condições ${ }^{(3)}$.

Estudos apontam as fragilidades das políticas públicas para a população em situação de rua e a sua vulnerabilidade em saúde, quando se vive em um ambiente que deveria ser de transitoriedade e não de permanência ${ }^{(1-4)}$.

Ademais, o viver em situação de rua está associado ao estigma e preconceito, independente das causas que levaram as pessoas a estarem nessa situação. Mesmo que seja transitória, grupos bastante heterogêneos, que estão distribuídos por diferentes faixas etárias; identidades; qualificações; realidades; demandas e problemas diversificados, vivem nos espaços públicos. No cenário de COVID-19, a questão torna-se ainda mais complexa e difícil de ser equacionada, quando não há informação oficial a respeito do número de pessoas vivendo na rua e as condições de saúde em que se encontram ${ }^{(5)}$.

A escassez ou a dificuldade de acesso aos serviços de saúde é uma cruel realidade a ser enfrentada pelos formuladores de políticas públicas e o Estado. São pessoas que, em muitos casos, não possuem documentos de registro civil; emprego formal; entre outras referências que os qualificam, segundo a normativa social para o acesso a bens e serviços da atenção social e saúde, entre outros, agravando potencialmente a invisibilidade e o risco desta população(3) ${ }^{(3)}$ Asim, torna-se imperativa a discussão acerca do tema.

\section{OBJETIVO}

O presente estudo se propôs a refletir acerca da vulnerabilidade e a invisibilidade da população que está vivendo em situação de rua, no contexto de pandemia de COVID-19.

\section{MÉTODOS}

Trata-se de um estudo teórico-reflexivo sobre a população brasileira em situação de rua durante a pandemia de COVID-19.

\section{VULNERABILIDADES DE PESSOAS EM SITUAÇÃO DE RUA E A COVID-19}

A pandemia não se restringe apenas aos problemas de saúde, mas ocasiona imensas implicações psicossociais que afetam toda a sociedade ${ }^{(6)}$. A vulnerabilidade é maior em indivíduos idosos, em pessoas com sistema imunológico deprimido; desnutridos; com doenças pulmonares pré-existentes e outras doenças sistêmicas ${ }^{(7)}$.

Alguns segmentos marginalizados, excluídos socialmente em situação de extrema pobreza e com condições de saúde fragilizadas, como os indivíduos em situação de rua, especialmente em Países com precárias e inadequadas medidas de saúde pública, podem ter uma combinação desses fatores, juntamente com estressores psicossociais ${ }^{(4,6)}$.

Trata-se de população com poucas ou nenhuma opção de fazer o auto isolamento de forma adequada e, menos ainda de ficar ou trabalhar em casa, carregando assim um fardo pesado de estresse e ansiedade, que pode estar ligado a qualquer combinação de fatores que vão desde altos níveis de estresse gerados pela insegurança em relação a ter ou não o alimento de cada dia e às moradias lotadas, ou inexistentes, vivenciando uma crise de saúde pública e, nesse caso, essa população é especialmente sensível às crises globais, como a atual pandemia de COVID-19(6). A população vivendo na rua representa grande desafio para os serviços sociais e de saúde. A falta de moradia está associada ao determinante social da saúde, mas raramente é visível e considerada entre as políticas públicas de saúde ${ }^{(4,6)}$.

As pessoas em situação de rua são extremamente vulneráveis ao contexto da atual pandemia, com potencial risco à exposição, aquisição e transmissão; com desproporcionalidades sociais afetadas pelas precárias condições 
de vida, saúde física e mental, associadas a problemas diversos e complexos, tais como, a superlotação dos locais onde vivem e dormem; falta de higiene; inadequado descarte de resíduos; ambientes com temperaturas extremas; contaminação; aumento da prevalência de infecções; uso e abuso substâncias; maior suscetibilidade a doenças físicas, sexuais, mentais e respiratórias. A prevalência de doenças respiratórias nessa população, geralmente é elevada, e, quando associadas à pandemia, as chances de agravamento da doença e morte se tornam ainda maiores ${ }^{(8)}$.

Os índices de mortalidade entre pessoas em situação de rua com menos de 65 anos, por todas as causas que é de 5-10 vezes maior do que a da população em geral ${ }^{(9)}$ e 20 vezes mais para tuberculose $\mathrm{e}^{(8)}$. Todavia, a infecção por COVID-19 pode aumentar ainda mais a disparidade de mortalidade neste grupo ${ }^{(4)}$.

Muitas pessoas em situação de rua têm doenças crônicas e problemas de saúde mental, com altos índices de abuso de substâncias psicoativas, possuem pouco acesso a serviços e cuidados de saúde, mediante a exigência de comprovantes de residências e documentos pessoais, deixando-os fora do sistema de assistência social, em alguns casos, e dificultando o acesso aos serviços, triagem, quarentena e tratamento quando há presença da COVID-19(4).

Por fim, destaca-se que, para o uso de qualquer tipo de intervenção junto a essa população, devem ser considerados todos os aspectos relacionados à vulnerabilidade e falta de alternativas seguras ao espaço público. Assim como a cooperação entre setores da assistência social, saúde, justiça e cidadania, por meio de medidas não coercitivas direcionadas, que possam ser implementadas para garantir que se conecte essas pessoas com um apoio seguro, ao invés de torná-las mais vulneráveis ${ }^{(8)}$.

\section{O ACESSO AOS SERVIÇOS DE SAÚDE POR PESSOAS QUE VIVEM EM SITUAÇÃO DE RUA}

O acesso da população em situação de rua à informação e à saúde é precário e de baixo alcance. Pessoas que vivem em situação de rua estão geograficamente em constante ir e vir pelo espaço urbano, caracterizando movimentos pendulares diários entre o local de alimentação, pernoite, descanso, que pode ser ou não o mesmo no decorrer do dia.

Os indivíduos que vivem em situação de rua e procuram abrigamento temporário ou pernoite em albergues também, estão desprotegidos. Geralmente, os abrigos trabalham acima da sua capacidade estrutural, em especial durante o inverno, há o compartilhamento de cobertores e banheiros insalubres, e o distanciamento das camas inferior a dois metros, que são alguns dos fatores que potencializam a transmissão, com especial atenção para os casos assintomáticos ${ }^{(10-12)}$.

Ademais, foi evidenciada a transmissão oro-fecal do vírus da COVID 19, associada a hábitos de higiene precários relacionados às necessidades básicas, entre esses indivíduos. A defecação muitas vezes ocorre em locais públicos, ambiente esse que também faz parte de sua alimentação e repouso. Essa instabilidade de "moradia" e a realização pública de tarefas da vida diária privadas, também contribuem para o risco de contaminação por esses indivíduos ${ }^{(4)}$.

É importante, pensando no contexto do viver na rua, citar a presença de imigrantes compondo essa população, a partir da onda de grandes migrações por conflitos e questões econômicas nos últimos anos. A situação da população de refugiados (imigrantes) vivendo nas ruas, se agrava com os desafios financeiros e a barreira do idioma. Tais desafios, associados à discriminação, por serem estrangeiros e as diferenças culturais, se apresentam como empecilhos importantes na procura por assistência em saúde ${ }^{(5)}$.

Em tempos de pandemia, viver em situação de rua aumenta consideravelmente o risco de contaminação; as dificuldades de acesso a serviços sociais, de saúde e a insumos básicos de higiene; que são necessários no dia a dia e obrigatórios no caso da prevenção à doença, comprometem a prevenção e o diagnóstico precoce, além do tratamento e controle de transmissão interpessoal do vírus.

Considerando o aumento do desemprego uma das consequências econômicas da pandemia, estima-se que haverá um aumento significativo de moradores de rua nas grandes cidades, o que ocasionará uma pressão súbita sobre o sistema de saúde e de assistência social, agravada pela perda do seguro assistencial de trabalhadores ${ }^{(10,12)}$. 
A violência institucional também tensiona o acesso aos serviços de saúde. Estigma e preconceito são expressos em agressões verbais e assédio moral, seja nos serviços de saúde ou nos abrigos, o que causa, subjetivamente, uma aversão a esses ambientes, agravando o processo de institucionalização do viver na rua. $O$ estigma social acentua a invisibilidade e exclusão social das pessoas sem moradia, acarretando baixo acompanhamento de saúde ${ }^{(13)}$.

Durante a pandemia, a dificuldade de acesso a serviços de abrigamento e saúde se intensifica. Algumas unidades de saúde foram destinadas emergencialmente para o atendimento de pacientes com suspeita de COVID 19, e os abrigos não estão aceitando novos usuários ${ }^{(12,13)}$. Assim, é fundamental que os profissionais orientem a população em situação de rua a respeito da adequação na rede de atenção à saúde e de assistência social durante a pandemia, direcionando a demanda em caso de situações crônicas ou de sintomatologia semelhante à da COVID ${ }^{(11,14)}$.

Em alguns Países foram criados novos serviços ou locais de atendimento específicos para a população que vive em situação de rua, durante a pandemia. A depender da sintomatologia quando infectado, o indivíduo não tem a necessidade de ficar internado, porém, precisa manter-se em isolamento ou distanciamento social, esses novos espaços consideram as particularidades da clínica com desabrigados.

Em Boston e em Nova York (EUA), foram implantadas tendas fásicas, que possibilitam o atendimento por etapas; níveis de gravidade e necessidade de cuidados, a saber: (1) pessoas assintomáticas sem exposição conhecida ao vírus que estão precisando de distanciamento físico; (2) pessoas assintomáticas com exposição conhecida ou suspeita ao vírus; (3) pessoas sintomáticas que aguardam resultados de exames, e (4) pacientes que têm diagnóstico confirmado do COVID-19(11).

Outra alternativa foi a utilização de quartos de hotéis que estão impedidos de funcionar durante a pandemia e dormitórios universitários para abrigamento, para privá-los de viver e estarem em situação na rua, durante a pandemia ${ }^{(10,15)}$.

Além disso, nesses abrigos em funcionamento e nas novas modalidades de moradias temporárias, são realizados os testes de identificação da COVID-19 a cada duas semanas para a população em situação de rua e para os trabalhadores, como forma de monitoramento e rastreamento de sintomas ${ }^{(10,11,15)}$.

A pandemia exacerbou uma situação grave de pouco investimento em saúde e assistência social às pessoas em situação de rua, escancarando a necessidade de enfrentamento da falta de moradia, por meio de ações multisetoriais e governamentais.

\section{Implicações para a Enfermagem}

A enfermagem tem sido protagonista no enfrentamento da pandemia, e tem a oportunidade de se reinventar no cuidado direcionado à população em situação de rua, desenvolvendo ações de prevenção em parcerias intersetoriais com a assistência social, segurança pública e educação, entre outras instâncias do poder público.

Em tempos de pandemia, os mais vulneráveis têm acesso limitado a cuidados de saúde, higiene, nutrição de qualidade e a recursos sociais de apoio. A falta de moradia apresenta vários desafios com níveis de complexidade que podem exacerbar e amplificar a expansão do COVID-19.

Estudo sugere que, desde a pandemia espanhola, os enfermeiros trabalham na linha de frente, realizando cuidados às populações vulneráveis afetadas pelo surto da doença. Nesse sentido, os profissionais da área da saúde, assim como representantes governamentais precisam rever as atuais estratégias de saúde, a fim de minimizar as vulnerabilidades dessa população(16).

As equipes de saúde, incluindo os enfermeiros, devem planejar fluxos de atendimento e medidas de prevenção com foco na melhor maneira de utilizar os recursos disponíveis para atender as diferentes demandas dessa população. Nos Estados Unidos (EU) estão sendo desenvolvidas ações para o estabelecimento de equipes e estruturas, que possam minimizar os riscos de disseminação da COVID-19, como por exemplo, promoção de práticas de controle de infecção em abrigos/albergues ${ }^{(16)}$. 
As equipes interdisciplinares e multiprofissionais são necessárias para minimizar os impactos da pandemia, em especial, na população em situação de rua, adaptando procedimentos operacionais padrão, e alinhando as orientações de cuidados primários para o COVID-19. Na Inglaterra, os enfermeiros trabalham em parceria com as equipes de apoio à habitação para levar serviços de saúde às pessoas abrigadas ${ }^{(10)}$.

Entre as alternativas apresentadas para manutenção dos atendimentos, de forma segura para profissionais e usuários, destacam-se as plataformas digitais, como a consulta por via telefônica. Aqueles cuidados que antes da pandemia eram realizados de forma presencial, mas que munidos de instruções poderiam ser realizados de forma individual, como a realização de curativos, por exemplo, estão sendo adaptados para que os acometidos desenvolvam a gestão do autocuidado. Ademais, outras formas de cuidado encontradas pelas equipes de enfermagem foram as distribuições de kits de higiene e alimentação, além de material informativo acerca da COVID-19(10).

No Reino Unido, os enfermeiros relataram a criação das acomodações de emergências, uma medida que contribuiu para minimizar a tensão entre a população em situação de rua, e que agora pode se ocupar de sua saúde e, ao mesmo tempo, ter acesso facilitado aos cuidados de saúde ${ }^{(10)}$. Estudo sinaliza que as experiências de cuidado vivenciadas por enfermeiros e outros profissionais de saúde, nos abrigos/albergues alternativos, podem oferecer modelos de cuidados inovadores para melhorar a prestação de cuidados e o envolvimento com os indivíduos em situação de rua ${ }^{(16)}$.

O reconhecimento da necessidade de investimento sistemático nos determinantes sociais e da saúde de pessoas que vivem nas ruas tem suas especificidades, tais como, a implementação de programas de apoio habitacionais e a assistência social e de saúde de forma sustentada.

\section{CONSIDERAÇÕES FINAIS}

Existem poucos estudos sobre o impacto da pandemia na população de rua em diferentes países, incluindo o Brasil, a escassez de pesquisas com essa população nesse momento pode estar associada a dificuldade e risco de coleta de dados quando o isolamento social se faz necessário. Os dados referentes à situação das pessoas vivendo nas ruas provavelmente serão conhecidos pós isolamento, entretanto é importante discutir, ainda que teoricamente, a situação no cenário da pandemia de Covid-19.

A população vivendo em situação de rua está exposta a diferentes graus de situações, entre elas a fragilidade das condições de saúde e da assistência ao cuidado. A exposição dessa população passa pela questão da invisibilidade dessa população nas grandes cidades. Embora vivenciado mundialmente, esta situação se apresenta com contornos dramáticos, quando não se tem um dado confiável em relação ao número de pessoas que vivem desta forma, como é o caso brasileiro.

O presente estudo oferece elementos que evidenciam a necessidade de estudo censitário nacional da população em situação de rua no Brasil e para que formuladores de políticas públicas desenvolvam estratégias inclusivas para esta população, aponta também a imprescindibilidade dos profissionais de saúde serem inventivos no que concerne aos cuidados básicos de saúde, com destaque para a prevenção de agravos destinada a essa população, reduzindo riscos e ampliando cuidados. Diante de um sistema de saúde que tem como princípio a universalidade, integralidade e equidade, pensando na estrutura do sistema, o local possível e que tem expertise para essa articulação e abordagem é a atenção básica.

$O$ viver na rua é uma questão associada aos determinantes sociais em saúde que tem impacto na qualidade de vida e no acesso a assistência, e está permeado por estigma e preconceito, o que o impele para a invisibilidade. A questão tem pouca margem de manobra diante da necessidade de ações de curto, médio e longo prazo, para que se evite a disseminação e elevada mortalidade entre as pessoas em situação de rua no Brasil e no mundo. 


\section{AGRADECIMENTO}

Ao Departamento de Enfermagem Psiquiátrica e Saúde Mental/Associação Brasileira de Enfermagem (DEPSM/ABEn).

\section{REFERÊNCIAS}

1. World Health Organization (WHO). Maintaining essential health services: operational guidance for the COVID-19 context Interim guidance [Internet]. Genebra; 2020 [cited 2020 jul 27]. Available from: https://apps.who.int/iris/bitstream/ handle/10665/332240/WHO-2019-nCoV-essential_health_services-2020.2-eng.pdf?sequence=1\&isAllowed=y

2. Gaetz S, Dej E, Richter T, Redman M (editors). The State of Homelessness in Canada 2016 [Internet]. Toronto (CA): Canadian Observatory on Homelessness Press; 2016 [cited 2020 jul 27]. Avaliable from: https://homelesshub.ca/sites/default/files/ SOHC16_final_200ct2016.pdf

3. Natalino MAC. Estimativa da população em situação de rua no Brasil [Internet]. Rio de Janeiro; 2016 [cited 2020 jul 27]. Avaliable from: http://repositorio.ipea.gov.br/bitstream/11058/7289/1/td_2246.pdf

4. Tsai J, Wilson M. COVID-19: A potential public health problem for homeless populations. The Lancet Public Health. 2020;5(4): E186-E187. doi:10.1016/ S2468-2667(20)30053-0

5. Wilson S, McCloughen A, Parr J, Jackson D. "If you are homeless you are welcome here": Social obligations for the homeless and socially disadvantaged. Journal of Clinical Nursing. 2019;28(15-16):2721-2723. doi: 10.1111/jocn.14851.

6. Banerjee D, Bhattacharya P. The hidden vulnerability of homelessness in the COVID-19 pandemic: Perspectives from India. International Journal of Social Psychiatry. 2020;1-4. doi: 10.1177/0020764020922890

7. Guo YR, Cao QD, Hong ZS, Tan YY, Chen SD, Jin HJ, et al. The origin, transmission and clinical therapies on coronavirus disease 2019 (COVID-19) outbreak -an update on the status. Military Medical Research. 2020;7(1), 1-10. doi: 10.1186/ s40779-020-00240-0

8. European Federation of National Organisations. COVID-19: "Staying home" Not an option for people experiencing homelessness. Bruxelas; 2020 [cited 2020 jul 27]. Avaliable from: https://www.feantsa.org/en/news/2020/03/18/ covid19-staying-home-not-an-option-for-people-experiencing-homelessness

9. Baggett TP, Hwang SW, O'Connell JJ, Porneala BC, Stringfellow EJ, Oray EJ, et al. Mortality among homeless adults in Boston: shifts in causes of death over a 15-year period. JAMA Intern Med. 2013;173:189-95. doi: 10.1001/ jamainternmed.2013.1604

10. Gaeta JM, Nueces DL, Munson DG, Barocas JA, Walsh KE. Case 21-2020: a 66-Year-Old Homeless Man With Covid-19. N Engl J Med. 2020; Jun 24;1-9. doi: 10.1056/NEJMcpc2002421

11. Mosites E, Parker EM, Clarke KEN, Gaeta JM, Bagget TP, Imbert E, et al. Assessment of SARS-CoV-2 infection prevalence in homeless shelters - four U.S Cities, MMWR Morb Mortal Wkly Rep. 2020;May 1;69(17):521-522. doi: 10.15585/mmwr.mm6917e1.

12. Moore EM, Takada S, Ijadi-Maghsoodi R, Gelbert L. Homelessness and Substance Use During the COVID-19 Pandemic: A Guide for Primary Care Providers [Internet]. USA: Opioid Responde Network; 2020 [cited 2020 jul 27]. Available from: https://opioidresponsenetwork.org/Homelessness\%20and\%20Substance\%20Use\%20During\%20COVID\%20 Resource.6.2.20.pdf

13. Fernandes MA, Pillon SC, Ibiapina ARS, Silva JS, Carvalho RJ, Passos BVS, et al. A complexa realidade do viver em situação de rua. In:Toledo MM(org). Ações de saúde e geração de conhecimento nas ciências médicas. Ponta Grossa (BR): Atena; 2020. 234p.

14. Doran KM, Cha S, Cho R, DiPietro B, Gelberg L, Kushel M. Housing as Health Care During and After the COVID-19 Crisis. COVID-19: Annals of Family Medicine. 2020. Preprint [cited 2020 Jul 02]. Available from: https://deepblue.lib.umich.edu/ bitstream/handle/2027.42/154767/Doran\%20main\%20article.pdf?sequence=1\&isAllowed=y

15. United States of America (USA). Covid-19 isolation site process, responsibilities, and best practices. Department of homeless services. NYC, 2020 [cited 2020 jul 27]. Available from: ttps://www.coalitionforthehomeless.org/wp-content/ uploads/2020/04/COVID19IsolationSite-Guidance_-as-of-040320.pdf

16. Dickins KA. Nurse's Perspective: COVID-19 and Homeless Populations. Mass General Research Institute. Boston, 2020 [cited 2020 jul 27]. Available from: https://mgriblog.org/2020/05/06/a-nurses-perspective-covid-19-and-homeless-populations/ 